\title{
HII Regions of NGC 628 and M101 as seen with SpIOMM
}

\author{
Laurie Rousseau-Nepton, Carmelle Robert, and Laurent Drissen \\ Université Laval \& CRAQ, Québec, QC, G1V 0A6, Canada \\ email: laurie.r-nepton.10ulaval.ca
}

\begin{abstract}
With SpIOMM, we obtained numerous spectra in the visible range covering simultaneously several emission lines of bright HII regions in the spiral galaxies NGC 628 and M101. We measured the size and luminosity of the HiI regions as well as the gas metallicity, temperature, and density. We estimated the age and star forming rate of the young stellar populations associated with the HiI regions. We looked for gradients along the galaxy radius and search for relations with the galactic arm positions. This is a first step in a project, based on a detailed study of stellar populations, to rebuild the history of spiral galaxies and to identify the mechanisms responsible for their evolution.
\end{abstract}

Keywords. Galaxies: spiral, galaxy: abundances, stars: formation, galaxies: evolution

\section{Introduction}

Many mechanisms over the history of a galaxy are considered to explain the compression of the gas responsible for bursts of star formation: feedback by stellar winds and supernovae, internal processes like spiral arm and bar, or external processes like galaxy interactions and gas inflow (e.g. Combes 2008). To understand the relative impact of these mechanisms, we undertook a study of the different stellar generations present in a sample of spiral galaxies seen face on. In this paper, the characteristics of the young stellar populations are presented based on a study of the visible spectrum of HII regions.

\section{Observations and Analysis}

Using SpIOMM, the Fourier transform spectrograph of the Observatoire du MontMégantic (see Drissen et al. 2011 for a description of the instrument), we observed NGC 628 (M74; SA(s)c) and M101 (NGC 5457 ; SAB(rs)cd). Two filters have been used over the wavebands 450 to $510 \mathrm{~nm}$ and 650 to $680 \mathrm{~nm}$, with a spectral resolution of 3 and $20 \AA$, respectively. SpIOMM offers a large field of view of $12^{\prime} \times 12^{\prime}$ with a spatial resolution of $65 \mathrm{pc} \mathrm{pix}^{-1}$ for NGC 628 (at a distance of $8.20 \mathrm{Mpc}$; NED) and $79 \mathrm{pc} \mathrm{pix}^{-1}$ for M101 (7.15 Mpc). Figure 1 shows, for example, the SpIOMM red continuum and $\mathrm{H} \alpha$ image of NGC 628 .

Using the SpIOMM H $\alpha+$ continuum image, we identified the brightest HiI regions with the program HIIphot of Thilker et al. (2000). We obtained 541 regions in NGC 628 and 715 regions in M101, which may be confined in groups of 5 to 25 pixels. Because of the limited spatial resolution, these regions may actually include more than a single HiI region. We separated the HiI regions in three dynamical groups: central regions are located within $2 \mathrm{kpc}$ from the galaxy center, arm regions are found in the $10 \%$ contour line over the $\mathrm{H} \alpha+$ continuum image, and the remaining HII regions are labeled disk regions. 


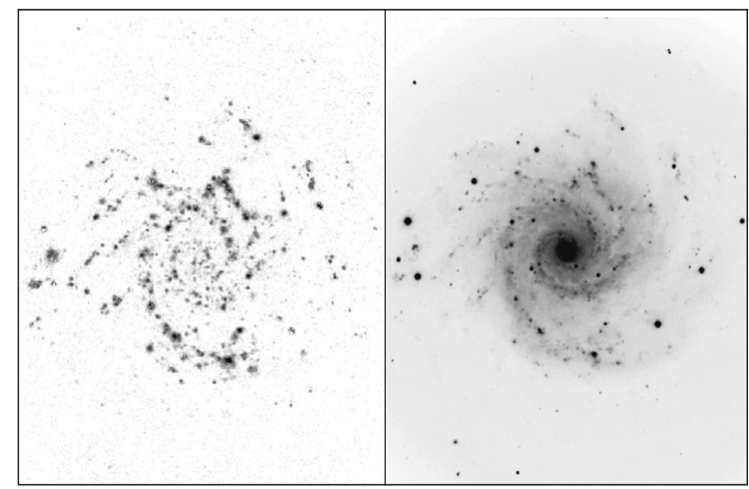

Figure 1. SpIOMM H $\alpha$ image (left) and red continuum image (right) of NGC 628.

\section{Results}

The star forming rate (SFR) is measured using the $\mathrm{H} \alpha$ luminosity and the equation of Kennicutt (1998). As shown in Figure 2 for NGC 628, a higher SFR may be seen for the more numerous arm regions. This is in good agreement with the work of Kennicutt et al. (1989) based directly on the $\mathrm{H} \alpha$ luminosity. The slope of $\mathrm{H} \alpha$ luminosity profiles for all the HII regions is also very consistent with the slope calculated by Kennicutt et al. (1989) for Sc type galaxies. For both galaxies we find that the number of disk regions is important. The SFR of the central regions, as for the disk regions, is also relatively small. As shown in Figure 3 for M101, we also find that the average $\mathrm{H} \alpha$ luminosity of the HII regions tend to increase (with a slope of 2.07) with the galactic radius.

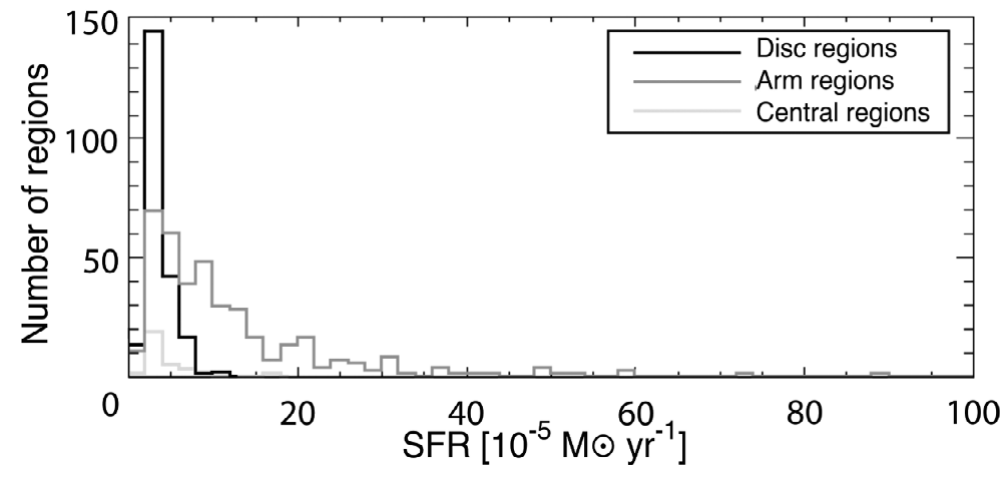

Figure 2. Star formation rate of the Hil regions in NGC 628.

We use the ratio $[\mathrm{NII}] \lambda 6583 / \mathrm{H} \alpha$ as a first indicator of the gas metallicity with the linear relation of Pettini \& Pagel (2004). The metallicity plots are shown in Figures 4 and 5 for the two galaxies. A mean value $12+\log [\mathrm{O} / \mathrm{H}]=8.6$ is obtained for both galaxies. This result is in relatively good agreement with the recent work of Sánchez et al. (2011) for NGC 628. A slightly larger value, $12+\log [\mathrm{O} / \mathrm{H}]=8.8$, is found for the central regions alone for both galaxies. A very small negative gradient is found, with a slope of -0.0053 for NGC 628 and -0.0044 for M101. There is no significant distinction of the average metallicity and slope between the arm and disk regions.

We present an histogram of the $\mathrm{H} \alpha$ equivalent width for NGC 628 HiI regions in Figure 6. These numbers along with the equivalent width of $\mathrm{H} \beta$ may be compared with models from Starburst99 (Leitherer et al. 1999) to obtain the age of the young stellar 


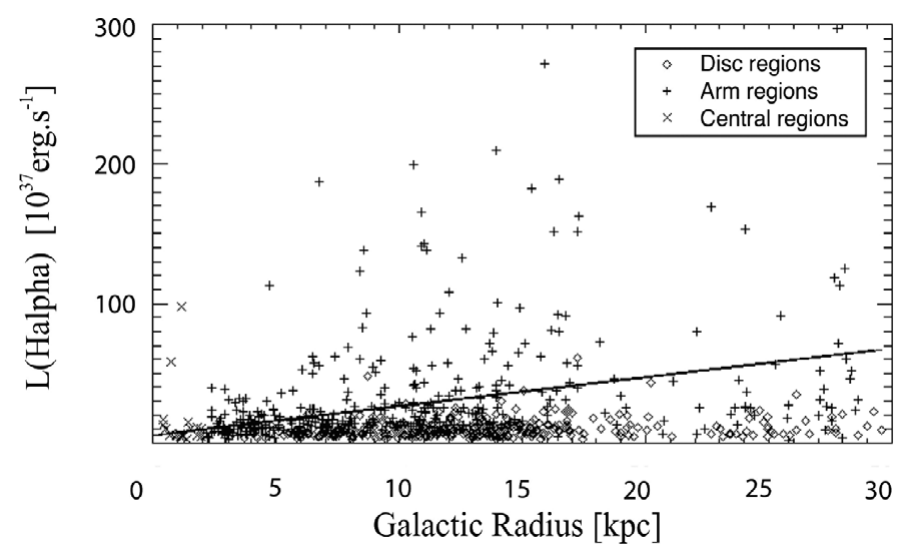

Figure 3. H $\alpha$ luminosity of the HiI regions in M 101 as a function of the galactic radius. The solid line is a linear fit through all the data points.

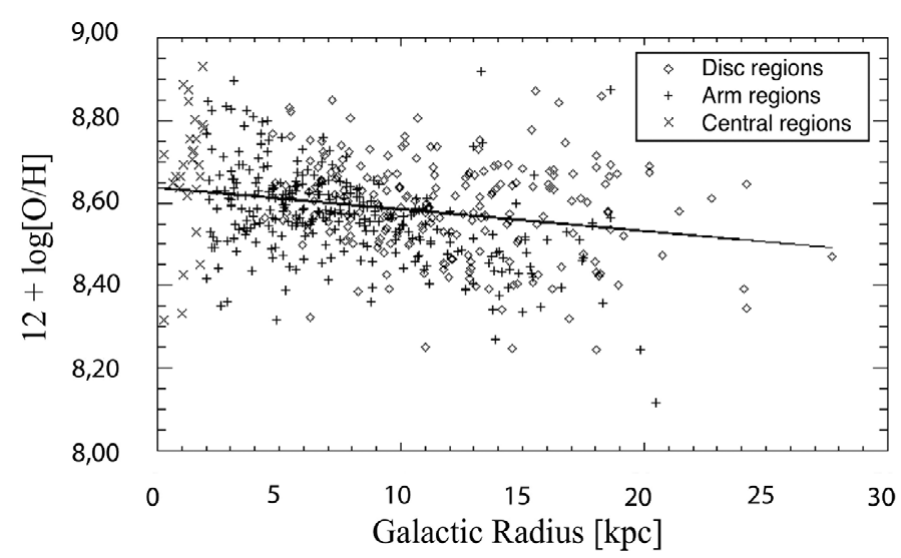

Figure 4. $[\mathrm{NII}] / \mathrm{H} \alpha$ metallicity for NGC 628 His regions as a function of the galactic radius. The solid line is a linear fit through all the data points.

population ionizing the gas within each regions. At this point, we see that larger values of the equivalent width, so younger stellar populations with an age between 3 and $6 \mathrm{Myr}$, are found in the arms. The average age of the disk regions is older, around $8 \mathrm{Myr}$.

\section{Discussion}

These preliminary results show the great potential of SpIOMM and integral-field units to study simultaneously the entire population of HiI regions in nearby galaxies. HiI regions may be counted and located within a galaxy. Their luminosity profile, stellar population, and metallicity gradient may be studied in details from their spectra with a large impact on our understanding of more distant galaxies and galaxy evolution.

This work is in progress. More data in the blue wavelength range around [OII] $\lambda 3727$ is expected soon with SpIOMM to refine the metallicity measurements along with a description of the ionization parameter. Long slit spectroscopy data have been collected over the arms, disk, and nucleus of these galaxies in the spectral range of 3500 to $8000 \AA$. It will be used to study the old underlying stellar populations. Visible and ultraviolet data are to be combined. Rotation field and internal gas flow will also be studied. A larger sample of nearby galaxies, including for example M63, NGC 6946, NGC 772 is being 


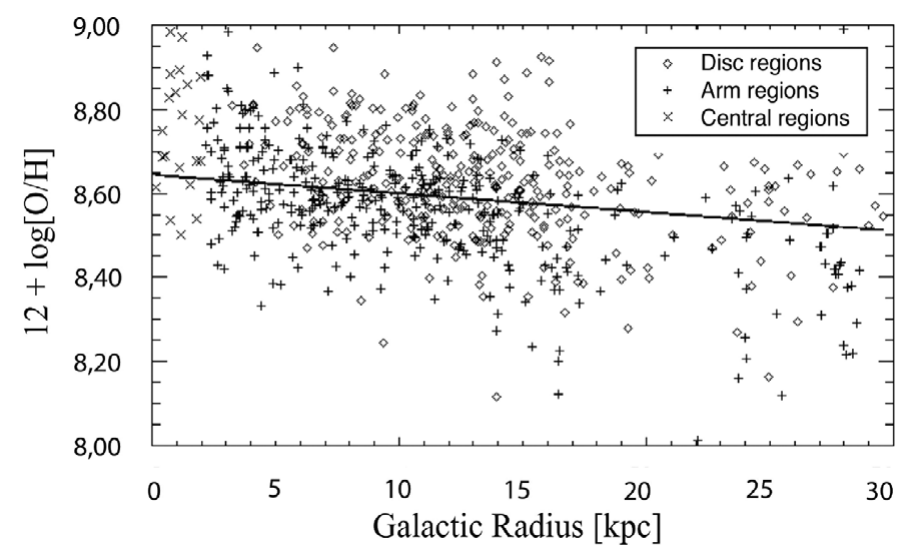

Figure 5. $[\mathrm{NII}] / \mathrm{H} \alpha$ metallicity for M101 Hit regions as a function of the galactic radius. The solid line is a linear fit through all the data points.

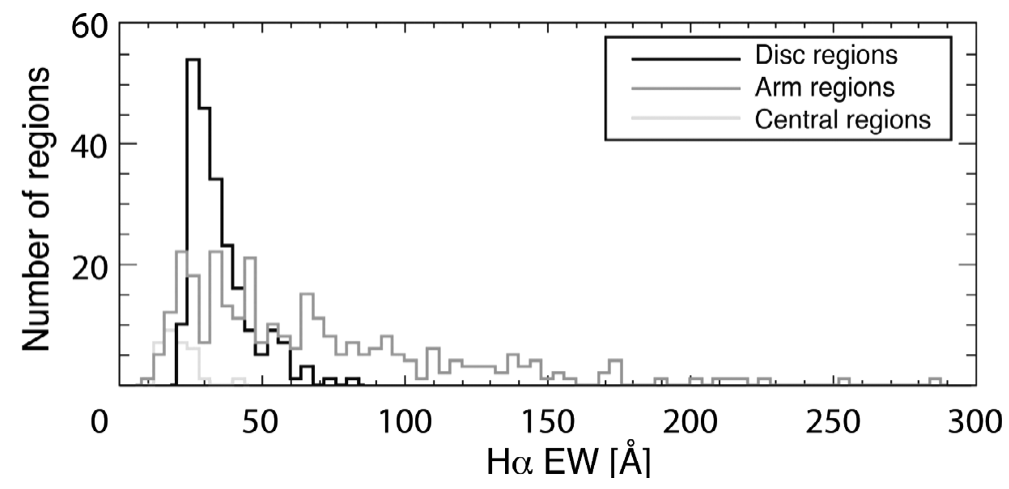

Figure 6. Equivalent width of the $\mathrm{H} \alpha$ emission line for NGC 628 Hit regions.

observed with SpIOMM in order to consider different galaxy properties (interaction, arms structures, bar, mass, etc.). A more refined definition of the arm HiI regions is to be considered to separate the inner and outer regions and see the impact of the different dynamics along the arm in relation, for example, with the different spiral arms models of Dobbs \& Pringle (2010). Better tests for the predictions of different scenarios for the star formation in spiral structures like interactions and gravitational waves and instabilities (e.g. Elmegreen 2011) are expected.

\section{References}

Combes, F. 2008, IAU Symp. 245, Formation and Evolution of Galaxy Bulges, 151

Dobbs, C. L. \& Pringle, J. E. 2010, MNRAS accepted

Drissen, L. \& Bernier, A.-P., et al. 2011, these proceedings

Elmegreen, B. G. 2011, arXiv:1101.3109v1

Kewley, L. J. \& Dopita, M. A. 2002, ApJS, 142, 35

Kennicutt, Jr., R. C. 1998, ARAA, 36, 189

Kennicutt, Jr., R. C., Edgar, B. K., \& Hodge, P. W. 1989, AJ, 337, 761

Leitherer, C., Schaerer, D., \& Goldader, et al. 1999, ApJS, 123, 3

Pettini, M. \& Pagel, B. E. J. 2004, MNRAS, 120, 3070

Sánchez, S. F., Rosales-Ortega, F. F., \& Kennicutt, R. C., et al. 2011, MNRAS accepted

Thilker, D. A., Braun, R., \& Walterbos, R. A. M. 2000, AJ, 348, L59 